\title{
Strategies for sequential prediction of stationary time series
}

\author{
László Györfi \\ Department of Computer Science and Information Theory \\ Technical University of Budapest \\ 1521 Stoczek u. 2, \\ Budapest, Hungary \\ gyorfi@szit.bme.hu \\ Gábor Lugosi * \\ Department of Economics, \\ Pompeu Fabra University \\ Ramon Trias Fargas 25-27, \\ 08005 Barcelona, Spain, \\ lugosi@upf .es
}

September 29, 2000

\section{Dedicated to the memory of Sid Yakowitz.}

\begin{abstract}
We present simple procedures for the prediction of a real valued sequence. The algorithms are based on a combination of several simple predictors. We show that if the sequence is a realization of a bounded stationary and ergodic random process then the average of squared errors converges, almost surely, to that of the optimum, given by the Bayes predictor. We offer an analog result for the prediction of stationary gaussian processes.
\end{abstract}

${ }^{*}$ The work of the second author was supported by DGES grant PB96-0300 


\section{Introduction}

One of the many themes of Sid's research was the search for prediction and estimation methods for time series that do not necessarily satisfy the classical assumptions for autoregressive markovian and gaussian processes (see, e.g., [17, 18, 26, 27, 28]). He firmly believed that most real-world applications require such robust methods. This note is a contribution to the line of research pursued and promoted by Sid who directed us to this beautiful area of research.

We study the problem of sequential prediction of a real valued sequence. At each time instant $i=1,2, \ldots$, the predictor is asked to guess the value of the next outcome $y_{i}$ of a sequence of real numbers $y_{1}, y_{2}, \ldots$ with knowledge of the past $y_{1}^{i-1}=\left(y_{1}, \ldots, y_{i-1}\right)$ (where $y_{1}^{0}$ denotes the empty string). Thus, the predictor's estimate, at time $i$, is based on the value of $y_{1}^{i-1}$. Formally, the strategy of the predictor is a sequence $g=\left\{g_{i}\right\}_{i=1}^{\infty}$ of decision functions

$$
g_{i}: \mathbb{R}^{i-1} \rightarrow \mathbb{R}
$$

and the prediction formed at time $i$ is $g_{i}\left(y_{1}^{i-1}\right)$. After $n$ rounds of play, the normalized cumulative prediction error on the string $y_{1}^{n}$ is

$$
L_{n}(g)=\frac{1}{n} \sum_{i=1}^{n}\left(g_{i}\left(y_{1}^{i-1}\right)-y_{i}\right)^{2}
$$

In this paper we assume that $y_{1}, y_{2}, \ldots$ are realizations of the random variables $Y_{1}, Y_{2}, \ldots$ drawn from the real valued stationary and ergodic process $\left\{Y_{n}\right\}_{-\infty}^{\infty}$. The fundamental limit for the predictability of the sequence can be determined based on a result of Algoet [2], who showed that for any prediction strategy $g$ and stationary ergodic process $\left\{Y_{n}\right\}_{-\infty}^{\infty}$,

$$
\liminf _{n \rightarrow \infty} L_{n}(g) \geq L^{*} \quad \text { almost surely, }
$$

where

$$
L^{*}=\mathbf{E}\left[\left(Y_{0}-\mathbf{E}\left\{Y_{0} \mid Y_{-\infty}^{-1}\right\}\right)^{2}\right]
$$

is the minimal mean squared error of any prediction for the value of $Y_{0}$ based on the infinite past $Y_{-\infty}^{-1}=\left(\ldots, Y_{-3}, Y_{-2}, Y_{-1}\right)$.

This lower bound gives sense to the following definition: 
Definition 1 A prediction strategy $g$ is called universal with respect to a class $\mathcal{C}$ of stationary and ergodic processes $\left\{Y_{n}\right\}_{-\infty}^{\infty}$, if for each process in the class,

$$
\lim _{n \rightarrow \infty} L_{n}(g)=L^{*} \quad \text { almost surely }
$$

Universal strategies asymptotically achieve the best possible loss for all ergodic processes in the class. Algoet [1] and Morvai, Yakowitz, Györfi [17] proved that there exists a prediction strategy universal with respect to the class of all bounded ergodic processes. However, the prediction strategies exhibited in these papers are either very complex or have an unreasonably slow rate of convergence even for well-behaved processes.

The purpose of this paper is to introduce several simple prediction strategies which, apart from having the above mentioned universal property of [1] and [17], promise much improved performance for "nice" processes. The algorithms build on a methodology worked out in recent years for prediction of individual sequences, see Vovk [29], Feder, Merhav, and Gutman [7], Littlestone and Warmuth [15], Cesa-Bianchi et al. [5], Kivinen and Warmuth [14], Singer and Feder [22], and Merhav and Feder [16] for a survey.

An approach similar to the one of this paper was adopted by Györfi, Lugosi, and Morvai [13], where prediction of stationary binary sequences was addressed. There we introduced a simple randomized predictor which predicts asymptotically as well as the optimal predictor for all binary ergodic processes. The present setup and results differ in several important points from those of [13]. On the one hand, special properties of the squared loss function considered here allow us to avoid randomization of the predictor, and to define a significantly simpler prediction scheme. On the other hand, possible unboundedness of a real-valued process requires special care, which we demonstrate on the example of gaussian processes. We refer to Nobel [19] to recent closely related work.

In Section 2 we introduce a universal strategy for bounded ergodic processes which is based on a combination of partitioning estimates. In Section 3 , still for bounded processes, we consider, as an alternative, a prediction strategy based on combining generalized linear estimates. In Section 4 we replace the boundedness assumption by assuming that the sequence to predict is an ergodic gaussian process, and show how the techniques of Section 3 may be modified to take care of the difficulties originating in the unboundedness of the process. 
The results of the paper are given in an autoregressive framework, that is, the value $Y_{t}$ is to be predicted based on past observations $Y_{1}^{t-1}$ of the same process. We may also consider the more general situation when $Y_{t}$ is predicted based on $Y_{1}^{t-1}$ and $X_{1}^{t}$, where $\left\{X_{n}\right\}_{-\infty}^{\infty}$ is an $\mathbb{R}^{d}$-valued process such that $\left\{\left(X_{n}, Y_{n}\right)\right\}_{-\infty}^{\infty}$ is a jointly stationary and ergodic process. The prediction problem is similar to the one defined above with the exception that the sequence of $X_{i}$ 's is also available to the predictor. One may think about the $X_{i}$ 's as side information. Formally, now a prediction strategy is a sequence $g=\left\{g_{i}\right\}_{i=1}^{\infty}$ of functions

$$
g_{i}: \mathbb{R}^{i-1} \times\left(\mathbb{R}^{d}\right)^{i} \rightarrow \mathbb{R}
$$

so that the prediction formed at time $i$ is $g_{i}\left(y_{1}^{i-1}, x_{1}^{i}\right)$. The normalized cumulative prediction error for any fixed pair of sequences $x_{1}^{n}, y_{1}^{n}$ is now

$$
L_{n}(g)=\frac{1}{n} \sum_{i=1}^{n}\left(g_{i}\left(y_{1}^{i-1}, x_{1}^{i}\right)-y_{i}\right)^{2} \text {, }
$$

All results of the paper may be extended, in a straightforward manner to this more general prediction problem. As the extension does not require new ideas, we omit the details.

Another direction for generalizing the results is to consider predicting vector-valued processes. Once again, the extension to $\mathbb{R}^{d}$-valued processes $\left\{Y_{n}\right\}_{-\infty}^{\infty}$ is obvious, and the details are omitted. 


\section{Universal prediction by partitioning esti- mates}

In this seqution we introduce our first prediction strategy for bounded ergodic processes. We assume throughout the section that $\left|Y_{0}\right|$ is bounded by a constant $B>0$, with probability one. First we assume that the bound $B$ is known. The case of unknown $B$ will be treated later in a remark.

The prediction strategy is defined, at each time instant, as a convex combination of elementary predictors, where the weighting coefficients depend on the past performance of each elementary predictor.

We define an infinite array of elementary predictors $h^{(k, \ell)}, k, \ell=1,2, \ldots$ as follows. Let $\mathcal{P}_{\ell}=\left\{A_{\ell, j}, j=1,2, \ldots, m_{\ell}\right\}$ be a sequence of finite partitions of the feature space $\mathbb{R}$, and let $G_{\ell}$ be the corresponding quantizer:

$$
G_{\ell}(x)=j, \text { if } x \in A_{\ell, j}
$$

With some abuse of notation, for any $n$ and $y_{1}^{n} \in \mathbb{R}^{n}$, we write $G_{\ell}\left(y_{1}^{n}\right)$ for the sequence $G_{\ell}\left(y_{1}\right), \ldots, G_{\ell}\left(y_{n}\right)$. Fix positive integers $k, \ell$, and for each $k$ long string $s$ of positive integers, define the partitioning regression function estimate

$$
\widehat{E}_{n}^{(k, \ell)}\left(y_{1}^{n-1}, s\right)=\frac{\sum_{\left\{k<i<n: G_{\ell}\left(y_{i-k}^{i-1}\right)=s\right\}} y_{i}}{\left|\left\{k<i<n: G_{\ell}\left(y_{i-k}^{i-1}\right)=s\right\}\right|}, \quad n>k+1,
$$

where $0 / 0$ is defined to be 0 .

Now we define the elementary predictor $h^{(k, \ell)}$ by

$$
h_{n}^{(k, \ell)}\left(y_{1}^{n-1}\right)=\widehat{E}_{n}^{(k, \ell)}\left(y_{1}^{n-1}, G_{\ell}\left(y_{n-k}^{n-1}\right)\right), \quad n=1,2, \ldots
$$

That is, $h_{n}^{(k, \ell)}$ quantizes the sequence $y_{1}^{n-1}$ according to the partition $\mathcal{P}_{\ell}$, and looks for all appearances of the last seen quantized strings $G_{\ell}\left(y_{n-k}^{n-1}\right)$ of length $k$ in the past. Then it predicts according to the average of the $y_{i}$ 's following the string.

The proposed prediction algorithm proceeds as follows: let $\left\{q_{k, \ell}\right\}$ be a probability distribution on the set of all pairs $(k, \ell)$ of positive integers such that for all $k, \ell, q_{k, \ell}>0$. Put $c=8 B^{2}$, and define the weights

$$
w_{t, k, \ell}=q_{k, \ell} e^{-(t-1) L_{t-1}\left(h^{(k, \ell)}\right) / c}
$$


and their normalized values

$$
v_{t, k, \ell}=\frac{w_{t, k, \ell}}{\sum_{i, j=1}^{\infty} w_{t, i, j}} .
$$

The prediction strategy $g$ is defined by

$$
g_{t}\left(y_{1}^{t-1}\right)=\sum_{k, \ell=1}^{\infty} v_{t, k, \ell} h^{(k, \ell)}\left(y_{1}^{t-1}\right), \quad t=1,2, \ldots
$$

Theorem 1 Assume that

(a) the sequence of partitions is nested, that is, any cell of $\mathcal{P}_{\ell+1}$ is a subset of a cell of $\mathcal{P}_{\ell}, \ell=1,2, \ldots$;

(b) if $\operatorname{diam}(A)=\sup _{x, y \in A}\|x-y\|$ denotes the diameter of a set, then for each sphere $S$ centered at the origin

$$
\lim _{\ell \rightarrow \infty} \max _{j: A_{\ell, j} \cap S \neq \emptyset} \operatorname{diam}\left(A_{\ell, j}\right)=0 .
$$

Then the prediction scheme $g$ defined above is universal with respect to the class of all ergodic processes such that $\mathbf{P}\left\{Y_{i} \in[-B, B]\right\}=1$.

One of the main ingredients of the proof is the following lemma, whose proof is a straightforward extension of standard arguments in the prediction theory of individual sequences, see, for example, Kivinen and Warmuth [14], Singer and Feder [23].

Lemma 1 Let $\tilde{h}_{1}, \tilde{h}_{2}, \ldots$ be a sequence of prediction strategies (experts), and let $\left\{q_{k}\right\}$ be a probability distribution on the set of positive integers. Assume that $\widetilde{h}_{i}\left(y_{1}^{n-1}\right) \in[-B, B]$ and $y_{1}^{n} \in[-B, B]^{n}$. Define

$$
w_{t, k}=q_{k} e^{-(t-1) L_{t-1}\left(\tilde{h}_{k}\right) / c}
$$

with $c \geq 8 B^{2}$, and

$$
v_{t, k}=\frac{w_{t, k}}{\sum_{i=1}^{\infty} w_{t, i}}
$$

If the prediction strategy $\tilde{g}$ is defined by

$$
\tilde{g}_{t}\left(y_{1}^{t-1}\right)=\sum_{k=1}^{\infty} v_{t, k} \tilde{h}_{k}\left(y_{1}^{t-1}\right) \quad t=1,2, \ldots
$$


then for every $n \geq 1$,

$$
L_{n}(\tilde{g}) \leq \inf _{k}\left(L_{n}\left(\tilde{h}_{k}\right)-\frac{c \ln q_{k}}{n}\right) .
$$

Here $-\ln 0$ is treated as $\infty$.

Proof. Introduce $W_{1}=1$ and $W_{t}=\sum_{k=1}^{\infty} w_{t, k}$ for $t>1$. First we show that for each $t>1$,

$$
\left[\sum_{k=1}^{\infty} v_{t, k}\left(y_{t}-\tilde{h}_{k}\left(y_{1}^{t-1}\right)\right)\right]^{2} \leq-c \ln \frac{W_{t+1}}{W_{t}}
$$

Note that

$$
W_{t+1}=\sum_{k=1}^{\infty} w_{t, k} e^{-\left(y_{t}-\tilde{h}_{k}\left(y_{1}^{t-1}\right)\right)^{2} / c}=W_{t} \sum_{k=1}^{\infty} v_{t, k} e^{-\left(y_{t}-\tilde{h}_{k}\left(y_{1}^{t-1}\right)\right)^{2} / c},
$$

so that

$$
-c \ln \frac{W_{t+1}}{W_{t}}=-c \ln \left(\sum_{k=1}^{\infty} v_{t, k} e^{-\left(y_{t}-\tilde{h}_{k}\left(y_{1}^{t-1}\right)\right)^{2} / c}\right) .
$$

Therefore, (2) becomes

$$
\exp \left(\frac{-1}{c}\left[\sum_{k=1}^{\infty} v_{t, k}\left(y_{t}-\tilde{h}_{k}\left(y_{1}^{t-1}\right)\right)\right]^{2}\right) \geq \sum_{k=1}^{\infty} v_{t, k} e^{-\left(y_{t}-\tilde{h}_{k}\left(y_{1}^{t-1}\right)\right)^{2} / c}
$$

which is implied by Jensen's inequality and the concavity of the function 


$$
\begin{aligned}
F_{t}(z)=e^{-\left(y_{t}-z\right)^{2} / c} \text { for } c \geq & 8 B^{2} \text {. Thus, (2) implies that } \\
n L_{n}(\tilde{g}) & =\sum_{t=1}^{n}\left(y_{t}-\tilde{g}\left(y_{1}^{t-1}\right)\right)^{2} \\
& =\sum_{t=1}^{n}\left[\sum_{k=1}^{\infty} v_{t, k}\left(y_{t}-\tilde{h}_{k}\left(y_{1}^{t-1}\right)\right)\right]^{2} \\
& \leq-c \sum_{t=1}^{n} \ln \frac{W_{t+1}}{W_{t}} \\
& =-c \ln W_{n+1} \\
& =-c \ln \left(\sum_{k=1}^{\infty} w_{n+1, k}\right) \\
& =-c \ln \left(\sum_{k=1}^{\infty} q_{k} e^{-n L_{n}\left(\tilde{h}_{k}\right) / c}\right) \\
& \leq-c \ln \left(\sup _{k} q_{k} e^{-n L_{n}\left(\tilde{h}_{k}\right) / c}\right) \\
& =\inf \left(-c \ln q_{k}+n L_{n}\left(\tilde{h}_{k}\right)\right)
\end{aligned}
$$

which concludes the proof.

Another main ingredient of the proof of Theorem 1 is known as Breiman's generalized ergodic theorem [4], see also Algoet [2].

Lemma 2 (BREIMAN [4]). Let $Z=\left\{Z_{i}\right\}_{-\infty}^{\infty}$ be a stationary and ergodic process. Let $T$ denote the left shift operator. Let $f_{i}$ be a sequence of realvalued functions such that for some function $f, f_{i}(Z) \rightarrow f(Z)$ almost surely. Assume that $\mathbf{E s u p}_{i}\left|f_{i}(Z)\right|<\infty$. Then

$$
\lim _{t \rightarrow \infty} \frac{1}{n} \sum_{i=1}^{n} f_{i}\left(T^{i} Z\right)=\mathbf{E} f(Z) \quad \text { almost surely. }
$$

Proof of Theorem 1. By a double application of the ergodic theorem, as 
$n \rightarrow \infty$, almost surely,

$$
\begin{aligned}
\widehat{E}_{n}^{(k, \ell)}\left(Y_{1}^{n-1}, s\right) & =\frac{\frac{1}{n} \sum_{\left\{k<i<n: G_{\ell}\left(Y_{i-k}^{i-1}\right)=s\right\}} Y_{i}}{\frac{1}{n}\left|\left\{k<i<n: G_{\ell}\left(Y_{i-k}^{i-1}\right)=s\right\}\right|} \\
& \rightarrow \frac{\mathbf{E}\left\{Y_{0} I_{\left\{G_{\ell}\left(Y_{-k}^{-1}\right)=s\right\}}\right\}}{\mathbf{P}\left\{G_{\ell}\left(Y_{-k}^{-1}\right)=s,\right\}} \\
& =\mathbf{E}\left\{Y_{0} \mid G_{\ell}\left(Y_{-k}^{-1}\right)=s\right\},
\end{aligned}
$$

and therefore

$$
\lim _{n \rightarrow \infty} \sup _{s}\left|\widehat{E}_{n}^{(k, \ell)}\left(Y_{1}^{n-1}, s\right)-\mathbf{E}\left\{Y_{0} \mid G_{\ell}\left(Y_{-k}^{-1}\right)=s\right\}\right|=0 \quad \text { almost surely. }
$$

Thus, by Lemma 2 , as $n \rightarrow \infty$, almost surely,

$$
\begin{aligned}
L_{n}\left(h^{(k, \ell)}\right) & =\frac{1}{n} \sum_{i=1}^{n}\left(h^{(k, \ell)}\left(Y_{1}^{i-1}\right)-Y_{i}\right)^{2} \\
& =\frac{1}{n} \sum_{i=1}^{n}\left(\widehat{E}_{n}^{(k, \ell)}\left(Y_{1}^{i-1}, Y_{i-k}^{i-1}\right)-Y_{i}\right)^{2} \\
& \rightarrow \mathbf{E}\left\{\left(Y_{0}-\mathbf{E}\left\{Y_{0} \mid G_{\ell}\left(Y_{-k}^{-1}\right)\right\}\right)^{2}\right\} \\
& \stackrel{\text { def }}{=} \epsilon_{k, \ell} .
\end{aligned}
$$

Since the partitions $\mathcal{P}_{\ell}$ are nested, $\mathbf{E}\left\{Y_{0} \mid \mathcal{G}_{\ell}\left(Y_{-k}^{-1}\right)\right\}$ is a martingale indexed by the pair $(k, \ell)$. Thus, the martingale convergence theorem (see, e.g., Stout [24]) and assumption (b) for the sequence of partitions implies that

$$
\lim _{k, \ell \rightarrow \infty} \epsilon_{k, \ell}=\mathbf{E}\left\{\left(Y_{0}-\mathbf{E}\left\{Y_{0} \mid Y_{-\infty}^{-1}\right\}\right)^{2}\right\}=L^{*}
$$

Now by Lemma 1,

$$
L_{n}(g) \leq \inf _{k, \ell}\left(L_{n}\left(h^{(k, \ell)}\right)-\frac{c \ln q_{k, \ell}}{n}\right),
$$


and therefore, almost surely,

$$
\begin{aligned}
\limsup _{n \rightarrow \infty} L_{n}(g) & \leq \operatorname{limsupinf}_{n \rightarrow \infty}\left(L_{n}\left(h^{(k, \ell)}\right)-\frac{c \ln q_{k, \ell}}{n}\right) \\
& \leq \inf _{k, \ell} \limsup _{n \rightarrow \infty}\left(L_{n}\left(h^{(k, \ell)}\right)-\frac{c \ln q_{k, \ell}}{n}\right) \\
& \leq \inf _{k, \ell} \limsup _{n \rightarrow \infty} L_{n}\left(h^{(k, \ell)}\right) \\
& =\inf _{k, \ell} \epsilon_{k, \ell} \\
& =\lim _{k, \ell \rightarrow \infty} \epsilon_{k, \ell} \\
& =L^{*}
\end{aligned}
$$

and the proof of the theorem is finished.

Theorem 1 shows that asymptotically, the predictor $g_{t}$ defined by (1) predicts as well as the optimal predictor given by the regression function $\mathbf{E}\left\{Y_{t} \mid Y_{-\infty}^{t-1}\right\}$. In fact, $G_{t}$ gives a good estimate of the regression function in the following sense:

Corollary 1 Under the conditions of Theorem 1

$$
\lim _{n \rightarrow \infty} \frac{1}{n} \sum_{i=1}^{n}\left(\mathbf{E}\left\{Y_{i} \mid Y_{-\infty}^{i-1}\right\}-g_{i}\left(Y_{1}^{i-1}\right)\right)^{2}=0 \quad \text { almost surely. }
$$

Proof. By Theorem 1,

$$
\lim _{n \rightarrow \infty} \frac{1}{n} \sum_{i=1}^{n}\left(Y_{i}-g_{i}\left(Y_{1}^{i-1}\right)\right)^{2}=L^{*} \quad \text { almost surely. }
$$

Consider the following decomposition:

$$
\begin{aligned}
\left(Y_{i}-g_{i}\left(Y_{1}^{i-1}\right)\right)^{2}= & \left(Y_{i}-\mathbf{E}\left\{Y_{i} \mid Y_{-\infty}^{i-1}\right\}\right)^{2} \\
& +2\left(Y_{i}-\mathbf{E}\left\{Y_{i} \mid Y_{-\infty}^{i-1}\right\}\right)\left(\mathbf{E}\left\{Y_{i} \mid Y_{-\infty}^{i-1}\right\}-g_{i}\left(Y_{1}^{i-1}\right)\right) \\
& +\left(\mathbf{E}\left\{Y_{i} \mid Y_{-\infty}^{i-1}\right\}-g_{i}\left(Y_{1}^{i-1}\right)\right)^{2}
\end{aligned}
$$

Then the ergodic theorem implies that

$$
\lim _{n \rightarrow \infty} \frac{1}{n} \sum_{i=1}^{n}\left(Y_{i}-\mathbf{E}\left\{Y_{i} \mid Y_{-\infty}^{i-1}\right\}\right)^{2}=L^{*} \quad \text { almost surely. }
$$


It remains to show that

$\lim _{n \rightarrow \infty} \frac{1}{n} \sum_{i=1}^{n}\left(Y_{i}-\mathbf{E}\left\{Y_{i} \mid Y_{-\infty}^{i-1}\right\}\right)\left(\mathbf{E}\left\{Y_{i} \mid Y_{-\infty}^{i-1}\right\}-g_{i}\left(Y_{1}^{i-1}\right)\right)=0 \quad$ almost surely

But this is a straightforward consequence of a classical srong law of large numbers for martingale differences, due to Kolmogorov, which states that it $\left\{Z_{i}\right\}$ be a martingale difference sequence with

$$
\sum_{n=1}^{\infty} \frac{\mathbf{E} Z_{n}^{2}}{n^{2}}<\infty
$$

then

$$
\lim _{n \rightarrow \infty} \frac{1}{n} \sum_{i=1}^{n} Z_{i}=0 \quad \text { almost surely. }
$$

Thus, (4) is implied by Kolmogorov's theorem since the martingale differences $Z_{i}=\left(Y_{i}-\mathbf{E}\left\{Y_{i} \mid Y_{-\infty}^{i-1}\right\}\right)\left(\mathbf{E}\left\{Y_{i} \mid Y_{-\infty}^{i-1}\right\}-g_{i}\left(Y_{1}^{i-1}\right)\right)$ are bounded by $4 B^{2}$.

Remark. UnKNOWN $B$. The prediction strategy studied in this section may be easily extended to the case when the process $\left\{Y_{n}\right\}_{-\infty}^{\infty}$ is bounded, but $B$ is unknown, that is, when no upper bound is known to the range of the process. In such a case we may simply start with the hypotheses $B=1$ and predict according to (1) until we find a value $Y_{n}$ with $\left|Y_{n}\right|>B$. Then we reset the algorithm and start the predictor again but with doubling the value of the previous $B$, and keep doing this. Then the universal property of Theorem 1 obviously remains valid to this modified strategy.

Remark. ChOICE of $q_{k, \ell}$. Theorem 1 is true independently of the choice of the $q_{k, \ell}$ 's as long as these values are strictly positive for all $k$ and $\ell$. In practice, however, the choice of $q_{k, \ell}$ may have an impact on the performance of the predictor. For example, if the distribution $\left\{q_{k, \ell}\right\}$ has a very rapidly decreasing tail, then the term $-\ln q_{k, \ell} / n$ will be large for moderetely large values of $k$ and $\ell$, and the performance of $g$ will be determined by the best of just a few of the elementary predictors $h^{(k, \ell)}$. Thus, it may be advantageous to choose $\left\{q_{k, \ell}\right\}$ to be a large-tailed distribution. For example, $q_{k, \ell}=c_{0} k^{-2} \ell^{-2}$ is a safe choice, where $c_{0}$ is an appropriate normalizing constant. 
Remark. Sequential guessing. If the process takes values from a finite set, one is often interested in the sequential guessing of $Y_{n}$ upon observing the past $Y_{1}^{n-1}$. Such a problem was investigated (among others) by Györfi, Lugosi, and Morvai in [13], where it was assumed that $Y_{n}$ takes one of two values: $Y_{n} \in\{0,1\}$. Sequential guessing is then formally defined by a sequence $f=\left\{f_{i}\right\}_{i=1}^{\infty}$ of decision functions

$$
f_{i}:\{0,1\}^{i-1} \rightarrow\{0,1\}
$$

and the guess formed at time $i$ is $f_{i}\left(Y_{1}^{i-1}\right)$. The normalized cumulative loss of guessing by $f$ on the string $Y_{1}^{n}$ is

$$
R_{1}^{n}(f)=\frac{1}{n} \sum_{i=1}^{n} I_{\left\{f_{i}\left(Y_{1}^{i-1}\right) \neq Y_{i}\right\}},
$$

where $I$ denotes the indicator function. Algoet [2] showed that for any guessing strategy $f$ and stationary ergodic binary process $\lim \inf _{n \rightarrow \infty} R_{1}^{n}(f) \geq R^{*}$ almost surely, where

$$
R^{*}=\mathbf{E}\left[\min \left(\mathbf{P}\left\{Y_{0}=1 \mid Y_{-\infty}^{-1}\right\}, \mathbf{P}\left\{Y_{0}=0 \mid Y_{-\infty}^{-1}\right\}\right)\right]
$$

is the minimal expected probability of error of guessing $Y_{0}$ based on the infinite past $Y_{-\infty}^{-1}$. The existence of a guessing scheme $f$ for which $\lim _{n \rightarrow \infty} R_{1}^{n}(f)=$ $R^{*}$ almost surely follows from results of Onrstein [20] and Bailey [3]. In [13] a simple guessing procedure was proposed with the same asymptotic guarantees and with a good finite-sample behavior for Markov processes. The disadvantade of the predictor given in [13] is that it requires randomization. Here we observe that with the help of predictors having the universal convergence property of Theorem 1 we may easily define a nonrandomized guessing scheme with the desired convergence properties. Given a prediction strategy

$$
g_{i}: \mathbb{R}^{i-1} \times\left(\mathbb{R}^{d}\right)^{i} \rightarrow \mathbb{R}
$$

for a binary process $\left\{Y_{n}\right\}$, we simply define the guessing scheme $f$ by the decision functions

$$
f_{i}\left(y_{1}^{i-1}\right)= \begin{cases}1 & \text { if } g_{i}\left(y_{1}^{i-1}\right) \geq 1 / 2 \\ 0 & \text { otherwise }\end{cases}
$$

Then we may use the properties of $g$ established in Corollary 1 to conclude that the guessing scheme $g$ defined above has an average number of mistakes 
$R_{1}^{n}(f)$ converging to the oprimum $R^{*}$ almost surely. Indeed, defining the decision, based on observing the infinite past $y_{-\infty}^{i-1}$, minimizing the probability of error of guessing $Y_{i}$ :

$$
f_{i}^{*}\left(y_{-\infty}^{i-1}\right)= \begin{cases}1 & \text { if } \mathbf{E}\left\{Y_{i} \mid Y_{-\infty}^{i-1}=y_{-\infty}^{i-1}\right\} \geq 1 / 2 \\ 0 & \text { otherwise }\end{cases}
$$

we may write

$$
\begin{aligned}
& \limsup _{n \rightarrow \infty} R_{1}^{n}(f)-R^{*} \\
& \quad=\limsup _{n \rightarrow \infty}\left(\frac{1}{n} \sum_{i=1}^{n} I_{\left\{f_{i}\left(Y_{1}^{i-1}\right) \neq Y_{i}\right\}}-\frac{1}{n} \sum_{i=1}^{n} \mathbf{P}\left\{f_{i}^{*}\left(Y_{-\infty}^{i-1}\right) \neq Y_{i} \mid Y_{-\infty}^{i-1}\right\}\right)
\end{aligned}
$$

(by the ergodic theorem)

$=\limsup _{n \rightarrow \infty}\left(\frac{1}{n} \sum_{i=1}^{n} \mathbf{P}\left\{f_{i}\left(Y_{1}^{i-1}\right) \neq Y_{i} \mid Y_{-\infty}^{i-1}\right\}-\frac{1}{n} \sum_{i=1}^{n} \mathbf{P}\left\{f_{i}^{*}\left(Y_{-\infty}^{i-1}\right) \neq Y_{i} \mid Y_{-\infty}^{i-1}\right\}\right)$

(by the martingale convergence theorem)

$$
\leq \limsup _{n \rightarrow \infty} \frac{2}{n} \sum_{i=1}^{n}\left|\mathbf{E}\left\{Y_{i} \mid Y_{-\infty}^{i-1}\right\}-g_{i}\left(Y_{1}^{i-1}\right)\right|
$$

(by Theorem 2.2 in [6])

$$
\begin{aligned}
& \leq \limsup _{n \rightarrow \infty} 2 \sqrt{\frac{1}{n} \sum_{i=1}^{n}\left|\mathbf{E}\left\{Y_{i} \mid Y_{-\infty}^{i-1}\right\}-g_{i}\left(Y_{1}^{i-1}\right)\right|^{2}} \\
& \rightarrow \quad 0 \quad \text { (by Corollary 1.) }
\end{aligned}
$$

Thus, any predictor with the universal property established in Theorem 1 may be converted, in a natural way, into a universal guessing scheme. An alternative proof of the same fact is given by Nobel [19]. 


\section{Universal prediction by generalized linear estimates}

This section is devoted to an alternative way of defining a universal predictor for the class of all bounded ergodic processes. Once again, we apply the method described by Lemma 1 to combine elementary predictors, but now, instead of partitioning-based predictors, we use elementary predictors which are generalized linear predictors. Once again, we consider bounded processes, and assume that a positive constant $B$ is known such that $\mathbf{P}\left\{\left|Y_{0}\right| \leq B\right\}=1$. (The case of unknown $B$ may be treated similarly as in Section 2.)

We define an infinite array of elementary predictors $h^{(k, \ell)}, k, \ell=1,2, \ldots$ as follows. Let $\left\{\phi_{j}^{(k)}\right\}_{j=1}^{\ell}$ be real-valued functions defined on $\mathbb{R}^{k}$. The elementary predictor $h^{(k, \ell)}$ generates a prediction of form

$$
\tilde{h}^{(k, \ell)}\left(Y_{1}^{n-1}\right)=\sum_{j=1}^{l} c_{n, j} \phi_{j}^{(k)}\left(Y_{n-k}^{n-1}\right)
$$

such that the coefficients $c_{n, j}$ are calculated based on the past observations $Y_{1}^{n-1}$. Before defining the coefficients, note that one is tempted to define the $c_{n, i}$ 's as the coefficients which minimize

$$
\sum_{i=k+1}^{n-1}\left(Y_{i}-\sum_{j=1}^{\ell} c_{j} \phi_{j}^{(k)}\left(Y_{i-k}^{i-1}\right)\right)^{2}
$$

if $n>k$, and the all 0 vector otherwise. However, even though the minimum always exists, it is not unique in general, and therefore the minimum is not well-defined. Instead, we define the coefficients by a standard recursive procedure as follows (see, e.g., Tsypkin [25], Györfi [12], Singer and Feder [23]). Introduce

$$
\begin{gathered}
X_{i}=\left(\phi_{1}^{(k)}\left(Y_{i-k}^{i-1}\right), \ldots \phi_{\ell}^{(k)}\left(Y_{i-k}^{i-1}\right)\right)^{T}, \\
A_{n}=\sum_{i=k+1}^{n-1} X_{i} X_{i}^{T}
\end{gathered}
$$

and

$$
M_{n}=\sum_{i=k+1}^{n-1} Y_{i} X_{i} .
$$


Let $\sigma$ be an arbitrary positive constant, put $B_{n}=A_{n}+\sigma I$, and define

$$
c_{n}=\left(c_{n, 1}, \ldots, c_{n, \ell}\right)=\left(A_{n}+\sigma I\right)^{-1} M_{n}=B_{n}^{-1} M_{n},
$$

It easy to see that the inverse can be calculated recursively by

$$
B_{n+1}^{-1}=B_{n}^{-1}-\frac{B_{n}^{-1} X_{n} X_{n}^{T} B_{n}^{-1}}{1+X_{n}^{T} B_{n}^{-1} X_{n}},
$$

which makes the calculation of $c_{n}$ easy.

\section{Theorem 2 Define}

$$
h_{n}^{(k, \ell)}\left(Y_{1}^{n-1}\right)= \begin{cases}B & \text { if } \tilde{h}^{(k, \ell)}\left(Y_{1}^{n-1}\right)>B \\ -B & \text { if } \tilde{h}^{(k, \ell)}\left(Y_{1}^{n-1}\right)<-B \quad n=1,2, \ldots \\ \tilde{h}^{(k, \ell)}\left(Y_{1}^{n-1}\right) & \text { otherwise }\end{cases}
$$

Suppose $\left|\phi_{j}^{(k)}\right| \leq 1$ and for any fixed $k$ the set

$$
\left\{\sum_{j=1}^{\ell} c_{j} \phi_{j}^{(k)} ; \quad\left(c_{1}, \ldots, c_{\ell}\right), \ell=1,2, \ldots\right\}
$$

is dense in $C\left([-B, B]^{k}\right)$. Define a prediction strategy by combining the elementary predictors $h^{(k, \ell)}$ given by (1). The obtained predictor is universal with respect to the class of all ergodic processes with $\mathbf{P}\left\{Y_{i} \in[-B, B]\right\}=1$.

Proof. Let $\bar{A}_{n}=\mathbf{E} A_{n}$, and let $\left(\lambda_{n, j}, \varphi_{n, j}\right) j=1,2, \ldots \ell$ be an eigensystem of $\bar{A}_{n}$ that is, $\left\{\varphi_{n, j}\right\}$ are orthogonal solutions of the equation

$$
\bar{A}_{n} \varphi_{n, j}=\lambda_{n, j} \varphi_{n, j}
$$

and

$$
\lambda_{n, 1} \geq \cdots \geq \lambda_{n, \ell} \geq 0 .
$$

Let $0 \leq \ell^{\prime} \leq \ell$ be the integer for which $\lambda_{n, j}>0$ if $j \leq \ell^{\prime}$ and $\lambda_{n, j}=0$ if $j>\ell^{\prime}$. Express the vector $\bar{M}_{n}=\mathbf{E} M_{n}$ as

$$
\bar{M}_{n}=\sum_{i=1}^{\ell} u_{j} \varphi_{n, j},
$$


and define

$$
c^{*}=\left(c_{1}^{*}, \ldots, c_{\ell}^{*}\right)=\sum_{j=1}^{\ell^{\prime}} \frac{u_{j}}{\lambda_{n, j}} \varphi_{n, j}
$$

(It is easy to see that the value of the vector $c^{*}$ is independent of $n$.) It is shown by Györfi [12] that

$$
\mathbf{E}\left\{\left(Y_{0}-\sum_{j=1}^{\ell} c_{j}^{*} \phi_{j}^{(k)}\left(Y_{-k}^{-1}\right)\right)^{2}\right\}=\min _{\left(c_{1}, \ldots, c_{\ell}\right)} \mathbf{E}\left\{\left(Y_{0}-\sum_{j=1}^{\ell} c_{j} \phi_{j}^{(k)}\left(Y_{-k}^{-1}\right)\right)^{2}\right\}
$$

and moreover

$$
\lim _{n \rightarrow \infty}\left(c_{n, 1}, \ldots, c_{n, \ell}\right)=\left(c_{1}^{*}, \ldots, c_{\ell}^{*}\right) \quad \text { almost surely }
$$

Also, observe that by the ergodic theorem, for any fixed $\left(c_{1}, \ldots, c_{\ell}\right)$,

$$
\lim _{n \rightarrow \infty} \frac{1}{n} \sum_{i=k+1}^{n}\left(Y_{i}-\sum_{j=1}^{\ell} c_{j} \phi_{j}^{(k)}\left(Y_{i-k}^{i-1}\right)\right)^{2}=\mathbf{E}\left\{\left(Y_{0}-\sum_{j=1}^{\ell} c_{j} \phi_{j}^{(k)}\left(Y_{-k}^{-1}\right)\right)^{2}\right\}
$$

Therefore by (6), (5) and Lemma 2

$$
\begin{aligned}
\lim _{n \rightarrow \infty} L_{n}\left(h^{(k, \ell)}\right) & =\lim _{n \rightarrow \infty} \frac{1}{n} \sum_{i=k+1}^{n}\left(Y_{i}-h^{(k, \ell)}\left(Y_{1}^{i-1}\right)\right)^{2} \\
& \leq \lim _{n \rightarrow \infty} \frac{1}{n} \sum_{i=k+1}^{n}\left(Y_{i}-\tilde{h}^{(k, \ell)}\left(Y_{1}^{i-1}\right)\right)^{2} \\
& =\lim _{n \rightarrow \infty} \frac{1}{n} \sum_{i=k+1}^{n}\left(Y_{i}-\sum_{j=1}^{\ell} c_{i, j} \phi_{j}^{(k)}\left(Y_{i-k}^{i-1}\right)\right)^{2} \\
& =\mathbf{E}\left\{\left(Y_{0}-\sum_{j=1}^{\ell} c_{j}^{*} \phi_{j}^{(k)}\left(Y_{-k}^{-1}\right)\right)^{2}\right\} \text { almost surely } \\
& =\epsilon_{k, \ell} .
\end{aligned}
$$


Next define the coefficient vector $\tilde{c}_{k}=\left(\tilde{c}_{k, 1}, \ldots, \tilde{c}_{k, \ell}\right)$ to be any vector which achieves the mimimum in

$$
\min _{\left(c_{1}, \ldots, c_{\ell}\right)} \mathbf{E}\left\{\left(\mathbf{E}\left\{Y_{0} \mid Y_{-k}^{-1}\right\}-\sum_{j=1}^{\ell} c_{j} \phi_{j}^{(k)}\left(Y_{-k}^{-1}\right)\right)^{2}\right\}
$$

Then

$$
\begin{aligned}
\epsilon_{k, \ell}= & \mathbf{E}\left\{\left(Y_{0}-\mathbf{E}\left\{Y_{0} \mid Y_{-\infty}^{-1}\right\}\right)^{2}\right\}+\mathbf{E}\left\{\left(\mathbf{E}\left\{Y_{0} \mid Y_{-\infty}^{-1}\right\}-\sum_{j=1}^{\ell} c_{j}^{*} \phi_{j}^{(k)}\left(Y_{-k}^{-1}\right)\right)^{2}\right\} \\
\leq & L^{*}+\mathbf{E}\left\{\left(\mathbf{E}\left\{Y_{0} \mid Y_{-\infty}^{-1}\right\}-\sum_{j=1}^{\ell} \tilde{c}_{k, j} \phi_{j}^{(k)}\left(Y_{-k}^{-1}\right)\right)^{2}\right\} \\
\leq & L^{*}+2 \mathbf{E}\left\{\left(\mathbf{E}\left\{Y_{0} \mid Y_{-\infty}^{-1}\right\}-\mathbf{E}\left\{Y_{0} \mid Y_{-k}^{-1}\right\}\right)^{2}\right\} \\
& +2 \mathbf{E}\left\{\left(\mathbf{E}\left\{Y_{0} \mid Y_{-k}^{-1}\right\}-\sum_{j=1}^{\ell} \tilde{c}_{k, j} \phi_{j}^{(k)}\left(Y_{-k}^{-1}\right)\right)^{2}\right\} \\
& \stackrel{\text { def }}{=} L^{*}+2 \delta_{k}+2 \gamma_{k, \ell} .
\end{aligned}
$$

It is immediate by the martingale convergence theorem that

$$
\lim _{k \rightarrow \infty} \delta_{k}=0
$$

On the other hand, by the denseness assumption of the theorem, for any fixed $k$,

$$
\lim _{\ell \rightarrow \infty} \gamma_{k, \ell}=\lim _{\ell \rightarrow \infty} \mathbf{E}\left\{\left(\mathbf{E}\left\{Y_{0} \mid Y_{-k}^{-1}\right\}-\sum_{j=1}^{\ell} \tilde{c}_{k, j} \phi_{j}^{(k)}\left(Y_{-k}^{-1}\right)\right)^{2}\right\}=0 .
$$

Thus, we conclude that

$$
\inf _{k, \ell} \epsilon_{k, \ell}=\lim _{k, \ell \rightarrow \infty} \epsilon_{k, \ell}=L^{*}
$$


Finally, by Lemma 1,

$$
\begin{aligned}
\limsup _{n \rightarrow \infty} L_{n}(g) & \leq \limsup _{n \rightarrow \infty} \inf _{k, \ell}\left(L_{n}\left(h^{(k, \ell)}\right)-\frac{c \ln q_{k, \ell}}{n}\right) \\
& \leq \inf _{k, \ell} \limsup _{n \rightarrow \infty}\left(L_{n}\left(h^{(k, \ell)}\right)-\frac{c \ln q_{k, \ell}}{n}\right) \\
& \leq \inf _{k, \ell} \limsup _{n \rightarrow \infty} L_{n}\left(h^{(k, \ell)}\right) \\
& =\inf _{k, \ell} \epsilon_{k, \ell} \\
& =L^{*},
\end{aligned}
$$

which concludes the proof.

Again, as in Corollary 1, we may compare the predictor directly to the regression function. By the same argument, we obtain the following result. The details are left to the reader.

Corollary 2 Under the conditions of Theorem 2

$$
\lim _{n \rightarrow \infty} \frac{1}{n} \sum_{i=1}^{n}\left(\mathbf{E}\left\{Y_{i} \mid Y_{-\infty}^{i-1}\right\}-g_{i}\left(Y_{1}^{i-1}\right)\right)^{2}=0 \quad \text { almost surely. }
$$




\section{Prediction of Gaussian processes}

Up to this point we have always assumed that the process to predict is bounded. This excludes some important unbounded processes such as gaussian processes. In this section we define a predictor which is universal with respect to the class of all stationary and ergodic gaussian processes. For gaussian processes the best predictor (i.e., the regression function) is linear, and therefore we may use the techniques of the previous section in the special case when $\phi_{j}^{(k)}\left(y_{1}^{k}\right)=y_{j}$. However, the unboundedness of the process introduces some additional difficulty. To handle it, we use bounded elementary predictors as before, but the bound is increased with $n$. Also, we need to modify the way of combining these elementary predictors.

The proposed predictor is based on a convex combination of linear predictors of different orders. For each $k=1,2, \ldots$ introduce

$$
h^{(k)}\left(y_{1}^{n-1}\right)=\sum_{j=1}^{k} c_{n, j} y_{n-j}
$$

where the vector $c_{n}=\left(c_{n, 1}, \ldots, c_{n, k}\right)$ of coefficients is calculated by the formula introduced in Section 3:

$$
c_{n}=\left(A_{n}+\sigma I\right)^{-1} M_{n},
$$

where $\sigma$ is a positive number, $A_{n}=\sum_{i=k+1}^{n-1} X_{i} X_{i}^{T}$ and $M_{n}=\sum_{i=k+1}^{n-1} Y_{i} X_{i}$ with $X_{i}=Y_{i-k}^{i-1}$ for $i>k$.

Introduce the notation

$$
L_{n}^{N}(g)=\frac{1}{N-n} \sum_{i=n+1}^{N}\left(g_{i}\left(y_{1}^{i-1}\right)-y_{i}\right)^{2} .
$$

Then the predictor $g$ is defined as follows: for all $m=0,1,2, \ldots$, if $n$ is such that $2^{m} \leq n<2^{m+1}$, then

$g_{n}\left(y_{1}^{n-1}\right)= \begin{cases}\sum_{k=1}^{\infty} v_{n, k}^{(m)} h^{(k)}\left(y_{1}^{n-1}\right) & \text { if }\left|\sum_{k=1}^{\infty} v_{n, k}^{(m)} h^{(k)}\left(y_{1}^{n-1}\right)\right| \leq \ln (n) \\ \ln (n) \operatorname{sgn}\left(\sum_{k=1}^{\infty} v_{n, k}^{(m)} h^{(k)}\left(y_{1}^{n-1}\right)\right) & \text { otherwise. }\end{cases}$

where

$$
v_{n, k}^{(m)}=\frac{w_{n, k}^{(m)}}{\sum_{k=1}^{\infty} w_{n, k}^{(m)}} \quad \text { and } \quad w_{n, k}^{(m)}=q_{k, m} e^{-\left(n-1-2^{m}\right) L_{2^{m}}^{n-1}\left(h^{(k)}\right) /\left(2 m^{2}\right)}
$$


with $q_{k, m}=2^{-(m+1)} I_{k \leq 2^{m+1}}$.

Thus, we divide the time instances into intervals of exponentially increasing length and, after initializing the predictor at the beginning of such an interval, we use a different way of combining the elementary predictors $h^{(k)}$ in each such segment. The reason for this is that to be able to combine elementary predictors as in Lemma 1, we need to make sure that the predictor as well as the outcome to predict is appropriately bounded. In our case this can be achieved based on Lemma 3 below which implies that with very large probability, the maximum of $n$ identically distributed normal random variables is at most of the order of $\sqrt{\log n}$.

Theorem 3 The prediction strategy $g$ defined above is universal with respect to the class of all stationary and ergodic zero-mean gaussian processes. Also,

$$
L_{n}(g) \leq \inf _{k \geq 1} L_{n}\left(h^{(k)}\right)+\frac{2\left(\log _{2} n+1\right)^{3}}{n}+O\left(\frac{1}{n}\right) \quad \text { almost surely }
$$

At a key point the proof uses the following well-known properties of gaussian random variables:

Lemma 3 (Pisier [21])). Let $Z_{1}, \ldots, Z_{n}$ be zero-mean gaussian random variables with $\mathbf{E}\left\{Z_{i}^{2}\right\}=\sigma^{2}, i=1, \ldots, n$. Then

$$
\mathbf{E}\left\{\max _{i \leq n}\left|Z_{i}\right|\right\} \leq \sigma \sqrt{2 \ln (2 n)}
$$

and for each $u>0$,

$$
\mathbf{P}\left\{\max _{i \leq n}\left|Z_{i}\right|-\mathbf{E}\left\{\max _{i \leq n}\left|Z_{i}\right|\right\}>u\right\} \leq e^{-u^{2} / 2 \sigma^{2}}
$$

Proof of Theorem 3. Lemma 3 implies, by taking $u=2 \sigma \sqrt{2 \ln (2 n)}$,

$$
\mathbf{P}\left\{\max _{i \leq n}\left|Y_{i}\right|>3 \sigma \sqrt{2 \ln (2 n)}\right\} \leq \frac{1}{(2 n)^{4}} .
$$

This implies, by the Borel-Cantelli lemma, that with probability one there exists a finite index $T^{\prime}$ such that for all $n>T^{\prime}, \max _{i \leq n}\left|Y_{i}\right| \leq 3 \sigma \sqrt{2 \ln (2 n)}$. 
Also, there exists a finite index $T$ such that for all $n>T, \max _{i \leq n}\left|Y_{i}\right| \leq \ln (n)$. Therefore, denoting $\bar{n}=2^{\left.\log _{2} n\right\rfloor+1}$, we may write

$$
\begin{aligned}
n L_{n}(g)= & \sum_{m=0}^{\left\lfloor\log _{2} n\right\rfloor-1} 2^{m} L_{2^{m}}^{2^{m+1}-1}(g)+(n-\bar{n} / 2+1) L_{\bar{n} / 2}^{n}(g) \\
\leq & 4 T \ln ^{2}(T+1)+\sum_{m=\left\lfloor\log _{2} T\right\rfloor}^{\left\lfloor\log _{2} n\right\rfloor-1} 2^{m} L_{2^{m}}^{2^{m+1}-1}(g)+(n-\bar{n} / 2+1) L_{\bar{n} / 2}^{n}(g) \\
& \left(\text { since } \max \left(\left|Y_{n}\right|,\left|g\left(Y_{1}^{n-1}\right)\right|\right) \leq \ln (T+1) \text { if } n \leq T\right) \\
\leq & 4 T \ln ^{2}(T+1)+\sum_{m=0}^{\left\lfloor\log _{2} n\right\rfloor-1} 2^{m} \inf \left(L_{2^{m}}^{2^{m+1}-1}\left(h^{(k)}\right)-\frac{2 m^{2} \ln q_{k, m}}{2^{m}}\right) \\
& +(n-\bar{n} / 2+1) \inf _{k \geq 1}\left(L_{\bar{n} / 2}^{n}\left(h^{(k)}\right)-\frac{2\left\lfloor\log _{2} n\right\rfloor^{2} \ln q_{k, m}}{n-\bar{n} / 2+1}\right)
\end{aligned}
$$

(by Lemma 1)

$$
\begin{aligned}
\leq & 4 T \ln ^{2}(T+1)+\inf _{k \geq 1}\left(\sum_{m=0}^{\left\lfloor\log _{2} n\right\rfloor-1} 2^{m} L_{2^{m}}^{2^{m+1}-1}\left(h^{(k)}\right)\right. \\
& \left.+(n-\bar{n} / 2+1) L_{\bar{n} / 2}^{n}\left(h^{(k)}\right)\right)+\sum_{m=0}^{\left\lfloor\log _{2} n\right\rfloor} \frac{2 m^{2}(m+1)}{2^{m}} \\
\leq & 4 T \ln ^{2}(T+1)+n \inf _{k \geq 1} L_{n}\left(h^{(k)}\right)+2\left(\log _{2} n+1\right)^{3} .
\end{aligned}
$$

In other words,

$$
L_{n}(g) \leq \inf _{k \geq 1} L_{n}\left(h^{(k)}\right)+\frac{2\left(\log _{2} n+1\right)^{3}}{n}+O\left(\frac{1}{n}\right) \quad \text { almost surely }
$$

This proves the second statement of the theorem. To prove the claimed universality property, it suffices to show that for all ergodic gaussian processes,

$$
\limsup _{n \rightarrow \infty} \inf _{k \geq 1} L_{n}\left(h^{(k)}\right)=L^{*}
$$


This can be done similarly to the proof of Theorem 2:

$$
\begin{aligned}
\lim _{n \rightarrow \infty} L_{n}\left(h^{(k)}\right) & =\lim _{n \rightarrow \infty} \frac{1}{n} \sum_{i=k+1}^{n}\left(Y_{i}-h^{(k)}\left(Y_{1}^{i-1}\right)\right)^{2} \\
& \leq \lim _{n \rightarrow \infty} \frac{1}{n} \sum_{i=k+1}^{n}\left(Y_{i}-\sum_{j=1}^{k} c_{i, j} Y_{i-j}\right)^{2} \\
& =\mathbf{E}\left\{\left(Y_{0}-\sum_{j=1}^{k} c_{j}^{*} Y_{-j}\right)^{2}\right\} \\
& \stackrel{\text { def }}{=} \epsilon_{k} .
\end{aligned}
$$

Define the coefficient vector $\left(\tilde{c}_{k, 1}, \ldots, \tilde{c}_{k, \ell}\right)$ such that it minimizes

$$
\min _{\left(c_{1}, \ldots, c_{\ell}\right)} \mathbf{E}\left\{\left(\mathbf{E}\left\{Y_{0} \mid Y_{-k}^{-1}\right\}-\sum_{j=1}^{k} c_{j} Y_{-j}\right)^{2}\right\} \text {. }
$$

(If the minimum is not unique, choose one arbitrarily.) Then

$$
\begin{aligned}
\epsilon_{k}= & \mathbf{E}\left\{\left(Y_{0}-\mathbf{E}\left\{Y_{0} \mid Y_{-\infty}^{-1}\right\}\right)^{2}\right\}+\mathbf{E}\left\{\left(\mathbf{E}\left\{Y_{0} \mid Y_{-\infty}^{-1}\right\}-\sum_{j=1}^{k} c_{j}^{*} Y_{-j}\right)^{2}\right\} \\
\leq & L^{*}+\mathbf{E}\left\{\left(\mathbf{E}\left\{Y_{0} \mid Y_{-\infty}^{-1}\right\}-\sum_{j=1}^{k} \tilde{c}_{k, j} Y_{-j}\right)^{2}\right\} \\
\leq & L^{*}+2 \mathbf{E}\left\{\left(\mathbf{E}\left\{Y_{0} \mid Y_{-\infty}^{-1}\right\}-\mathbf{E}\left\{Y_{0} \mid Y_{-k}^{-1}\right\}\right)^{2}\right\} \\
& +2 \mathbf{E}\left\{\left(\mathbf{E}\left\{Y_{0} \mid Y_{-k}^{-1}\right\}-\sum_{j=1}^{k} \tilde{c}_{k, j} Y_{-j}\right)^{2}\right\} \\
= & L^{*}+2 \delta_{k}
\end{aligned}
$$

since

$$
\mathbf{E}\left\{\left(\mathbf{E}\left\{Y_{0} \mid Y_{-k}^{-1}\right\}-\sum_{j=1}^{k} \tilde{c}_{k, j} Y_{-j}\right)^{2}\right\}=0 .
$$

Now the proof can be finished by mimicking the proof of Theorem 2 .

Once again, we may derive a property analogous to Corollary 1: 
Corollary 3 Under the conditions of Theorem 3,

$$
\lim _{n \rightarrow \infty} \frac{1}{n} \sum_{i=1}^{n}\left(\mathbf{E}\left\{Y_{i} \mid Y_{-\infty}^{i-1}\right\}-g_{i}\left(Y_{1}^{i-1}\right)\right)^{2}=0 \quad \text { almost surely. }
$$

Proof. We proceed exactly as in the proof of Corollary 1 . The only thing that needs a bit more care is checking the conditions of Kolmogorov's strong law for sums of martingale differences, since in the gaussian case the corresponding martingale differences are not bounded. By the CauchySchwarz inequality,

$$
\begin{aligned}
\mathbf{E} Z_{i}^{2} & =\mathbf{E}\left\{\left(Y_{i}-\mathbf{E}\left\{Y_{i} \mid Y_{-\infty}^{i-1}\right\}\right)^{2}\left(\mathbf{E}\left\{Y_{i} \mid Y_{-\infty}^{i-1}\right\}-g_{i}\left(Y_{1}^{i-1}\right)\right)^{2}\right\} \\
& \leq \sqrt{\mathbf{E}\left\{\left(Y_{i}-\mathbf{E}\left\{Y_{i} \mid Y_{-\infty}^{i-1}\right\}\right)^{4}\right\} \mathbf{E}\left\{\left(\mathbf{E}\left\{Y_{i} \mid Y_{-\infty}^{i-1}\right\}-g_{i}\left(Y_{1}^{i-1}\right)\right)^{4}\right\}} \\
& \leq \sqrt{8 \mathbf{E}\left\{Y_{i}^{4}\right\}\left(\mathbf{E}\left\{Y_{i}^{4}\right\}+\mathbf{E}\left\{g_{i}\left(Y_{1}^{i-1}\right)^{4}\right\}\right)} \\
& \leq C \sqrt{\left(1+(\log i)^{4}\right)} \\
& \leq C^{\prime}(\log i)^{2}
\end{aligned}
$$

where $C$ and $C^{\prime}$ are positive constants, which implies $\sum_{i=1}^{\infty} \mathbf{E} Z_{i}^{2} / i^{2}<\infty$, so the condition of Kolmogorov's theorem is satisfied.

Remark. Rates of convergence. The inequality of Theorem 3 shows that the rate of convergence of $L_{n}(g)$ to $L^{*}$ is determined by the performance of the best elementary predictor $h^{(k)}$. The price of adaptation to the best elementary predictor is merely an additional term of the order of $n^{-1} \log ^{3} n$. This additional term is not much larger than an inevitable estimation error. This is supported by a result of Gerencsér and Rissanen [10] who showed that for any gaussian $\operatorname{ARMA}(p+q)$ process and for any predictor $g$,

$$
\mathbf{E} L_{n}(g) \geq L^{*}+(1-o(1)) L^{*}(p+q) \frac{\ln n}{n} .
$$

On the other hand, Gerencsér [9] showed under some mixing conditions for $\operatorname{ARMA}(p, q)$ processes that there exists a predictor $g_{p, q}$ such that

$$
L_{n}\left(g_{p, q}\right)=L^{*}+(1+o(1)) L^{*}(p+q) \frac{\ln n}{n} \quad \text { almost surely. }
$$


Further rate-of-convergence results under more general conditions for the process were established by Gerencsér [8]. Another general branch of bounds can be found in Goldenshluger and Zeevi [11]. Consider the MA( $\infty)$ representation of $Y_{n}$

$$
Y_{n}=\sum_{i=1}^{\infty} \psi_{i} \epsilon_{n-i}
$$

with transfer function

$$
\psi(z)=\sum_{i=1}^{\infty} \psi_{i} z^{i}
$$

Goldenshluger and Zeevi show that if for $0<l<1<L<\infty$ and $\rho>1$

$$
l<|\psi(z)|<L, \text { for }|z|<\rho
$$

then for large $n$

$$
\mathbf{E} L_{n}\left(h^{(k)}\right) \leq L^{*}+c(l, L) \frac{1}{n} \sum_{i=1}^{n}\left(\frac{1}{i^{2}(\rho-1) 2}+\frac{k}{\rho^{2 k}(\rho-1) 2}+\frac{k}{i}\right)
$$

and for $k=k_{n}=\left\lfloor\frac{\ln n}{2 \ln \rho}\right\rfloor$

$$
\mathbf{E} L_{n}\left(h^{(k)}\right) \leq L^{*}+C(l, L, \rho) \frac{1}{n} \sum_{i=1}^{n} \frac{\ln i}{i} \leq L^{*}+C(l, L, \rho) \frac{(\ln n)^{2}}{n} .
$$

Thus, for the processes investigated by Goldenshluger and Zeevi, the predictor $g$ of Theorem 3 achieves the rate of convergence

$$
\mathbf{E} L_{n}(g) \leq L^{*}+O\left(\frac{\log ^{3} n}{n}\right)
$$




\section{References}

[1] P. Algoet. Universal schemes for prediction, gambling, and portfolio selection. Annals of Probability, 20:901-941, 1992.

[2] P. Algoet. The strong law of large numbers for sequential decisions under uncertainity. IEEE Transactions on Information Theory, 40:609634, 1994.

[3] D.H. Bailey. Sequential schemes for classifying and predicting ergodic processes. PhD thesis, Stanford University, 1976.

[4] L. Breiman. The individual ergodic theorem of information theorey. Annals of Mathematical Statistics, 31:809-811, 1957. Correction. Annals of Mathematical Statistics, 31:809-810, 1960.

[5] N. Cesa-Bianchi, Y. Freund, D.P. Helmbold, D. Haussler, R. Schapire, and M.K. Warmuth. How to use expert advice. Journal of the ACM, 44(3):427-485, 1997.

[6] L. Devroye, L. Györfi, and G. Lugosi. A Probabilistic Theory of Pattern Recognition. Springer-Verlag, New York, 1996.

[7] M. Feder, N. Merhav, and M. Gutman. Universal prediction of individual sequences. IEEE Transactions on Information Theory, 38:1258$1270,1992$.

[8] L. Gerencsér. $A R(\infty)$ estimation and nonparametric stochastic complexity. IEEE Transactions on Information Theory, 38:1768-1779, 1992.

[9] L. Gerencsér. On Rissanen's predictive stochastic complexity for stationary ARMA processes. J. of Statistical Planning and Inference, 41:303$325,1994$.

[10] L. Gerencsér, and J. Rissanen. A prediction bound for Gaussian ARMA processes. Proc. of the 25th Conference on Decision and Control, 14871490, 1986.

[11] A. Goldenshluger, and A. Zeevi. Non-asymptotic bounds for autoregressive time series modeling. (submitted for publication). 
[12] L. Györfi. Adaptive linear procedures under general conditions. IEEE Transactions on Information Theory, 30:262-267, 1984.

[13] L. Györfi, G. Lugosi, and G. Morvai. A simple randomized algorithm for consistent sequential prediction of ergodic time series. IEEE Transactions on Information Theory, 45:2642-2650, 1999.

[14] J. Kivinen and M. K. Warmuth. Averaging expert predictions. In H. U. Simon P. Fischer, editor, Computational Learning Theory: Proceedings of the Fourth European Conference, EuroCOLT'99, pages 153167. Springer, Berlin, 1999. Lecture Notes in Artificial Intelligence 1572.

[15] N. Littlestone and M. K. Warmuth. The weighted majority algorithm. Information and Computation, 108:212-261, 1994.

[16] N. Merhav and M. Feder. Universal prediction. IEEE Transactions on Information Theory, 44:2124-2147, 1998.

[17] G. Morvai, S. Yakowitz, and L. Györfi. Nonparametric inference for ergodic, stationary time series. Annals of Statistics, 24:370-379, 1996.

[18] G. Morvai, S. Yakowitz, and P. Algoet. Weakly Convergent Stationary Time Series. IEEE Transactions on Information Theory, 43:483-498, 1997.

[19] A. Nobel. Aggregate schemes for sequential prediction of ergodic processes. manuscript, 2000.

[20] D.S. Ornstein. Guessing the next output of a stationary process. Israel Journal of Mathematics, 30:292-296, 1978.

[21] G. Pisier. Probabilistic methods in the geometry of Banach spaces. In Probability and Analysis. Lecture Notes in Mathematics, 1206, pages 167-241. Springer, New York, 1986.

[22] A. Singer and M. Feder. Universal linear prediction by model order weighting. IEEE Transactions on Signal Processing, 47:2685-2699, 1999.

[23] A. C. Singer, and M. Feder. Universal linear least-squares prediction. International Symposium of Information Theory, 2000.

[24] W.F. Stout. Almost sure convergence. Academic Press, New York, 1974. 
[25] Ya.Z. Tsypkin. Adaptation and Learning in Automatic Systems. Academic Press, New York, 1971.

[26] S. Yakowitz. Nearest-neighbour methods for time series analysis. Journal of Time Series Analysis, 8:235-247, 1987.

[27] S. Yakowitz. Nonparametric density and regression estimation for Markov sequences without mixing assumptions. Journal of Multivariate Analysis, 30:124-136, 1989.

[28] S. Yakowitz, L. Györfi, J. Kieffer, and G. Morvai. Strongly consistent nonparametric estimation of smooth regression functions for stationary ergodic sequences. Journal of Multivariate Analysis, 71:24-41, 1999.

[29] V.G. Vovk. Aggregating strategies. In Proceedings of the Third Annual Workshop on Computational Learning Theory, pages 372-383. Association of Computing Machinery, New York, 1990. 